\title{
ABC Spotlight on carbon nanotubes (CNTs)
}

\author{
Günter Gauglitz
}

Received: 7 July 2014 / Accepted: 7 July 2014 / Published online: 24 July 2014

(C) Springer-Verlag Berlin Heidelberg 2014

For many decades, nanomaterials have been the focus of research and applications. Their specific properties, such as surface-to-volume ratio, porosity, or antibacterial activity and plasmonics, provide many interesting applications in the fields of food stabilization, packaging materials, carriers for drugs, signal enhancement, chromatography and even sensing. Toxicity is also discussed, depending on the form, size and coating. For this reason, a high number of publications can be found that weigh the pros and cons of such nanomaterials. Examples include carbon nanotubes (CNTs) or carbon nanowires, which have attracted interest from the scientific community since the first reports in around 1991. At SpringerLink, more than 300 publications can be found that deal with CNTs, with the number of publications having increased in recent years. Accordingly, Analytical and Bioanalytical Chemistry is keeping track of this type of material and its application in the area of analytical chemistry. Future applications to analytical sciences were first discussed in 2005 [1]. In the beginning, the focus was on separation techniques, where single-, double- or multi-walled CNTs were used in various applications in the area of solid-phase micro-extraction or as modified stationary phases in gas chromatography or even in electrophoresis $[2,3]$. According to their structure, linear, bamboo and coiled CNTs are also identified. In particular, coiled structures obtained by incorporating pentagons or heptagons in the hexagonal sheets of CNTs in a periodic way open up interesting possibilities for applications in the areas of nanocomposites, nanoelectronic devices and nanoelectromechanical systems; these all provide possible interesting applications in sorption, extraction and

G. Gauglitz ( $\bowtie)$

Institute of Physical and Theoretical Chemistry, Eberhard Karls Universität, Auf der Morgenstelle 18, 72076 Tübingen, Germany

e-mail: abc.gg@ipc.uni-tuebingen.de chromatographic processes. In one of the recent publications [4], through the combination of micro-walled CNTs, solid-phase extraction and gas chromatography, the detection and quantification of pesticides in matrices, such as baby food, are improved; thus reducing the extraction time and simplifying the extraction procedure compared with conventional solid-phase extraction, and opening up new ways to reduce the limits of quantification, according to European Union residue limits in nutrition. These CNTs are also successfully used in micro-GC chips, because the single-walled CNT (SWCNT) stationary phase has good separation abilities. Examples of possibilities of functionalization are given in [5] to influence sorption capabilities (Fig. 1).

The use of CNTs as a drug-delivery system is also interesting and is opening up areas of nanomedicine. They are considered to help, in particular, in cancer diagnostics and are even effective in the treatment of diseases. CNTs can overcome problems with penetration of the cell membrane as a successful tool in modern healthcare. However, a recent publication balanced the advantages and disadvantages of using CNTs in biological applications, which are of highest priority in today's clinical medicine, such as in disease treatment and regenerative medicine. These advantages are compared with problems of current research for in vitro toxicity of CNTs for biomaterials and safety evaluation. More data have to be determined and regulations have to be introduced to reduce risks that arise during drug delivery and whole-body imaging, as well as effects on the heart, lung, liver, spleen, kidney and other organs. Pros, cons and future requirements are discussed in detail in [6]. Finding the correct balance requires a deep understanding of processes at and with these particles. Furthermore, intensive characterisation of the CNTs themselves, as well as their modification by ligands or marker molecules [7] is essential. Surface-enhanced Raman spectroscopy (SERS) 
Fig. 1 Examples of different possibilities of functionalization of CNTs. (a) Noncovalent exohedral functionalization with polymers. (b) Defect-group functionalization. (c) Noncovalent exohedral functionalization with molecules through p-stacking. (d) Sidewall functionalization. (e) Endohedral functionalization of, in this case, SWNT [5]

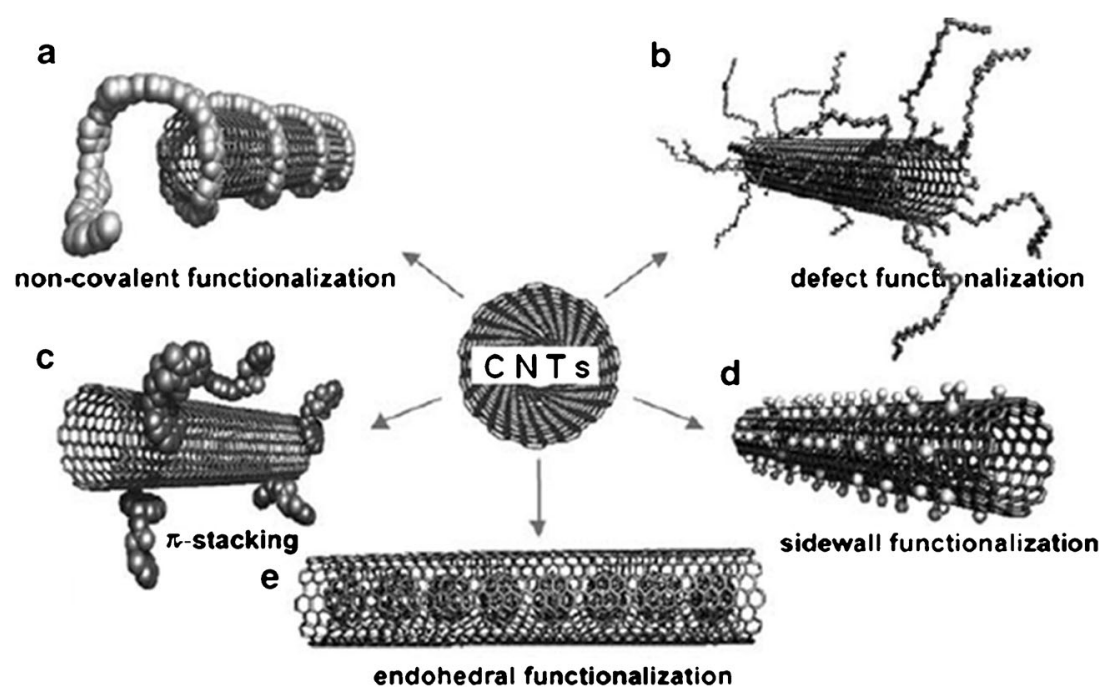

[8] is a well-known technique and an attractive tool to examine molecules in trace amounts in chemical and biochemical analytics. In addition, plasmonics of metal nanoparticles can enhance Raman spectroscopy; this means that the technique can be used to examine, in detail, the surface of CNTs or take advantage of localised information in combination with plasmonic structure.

For a few years, CNTs have also been used as recognition elements in electrochemical and optical sensing. Functionalised SWCNTs provide distinctive redox peaks for electrochemical detection. In combination with aptamers, they have been successfully applied to the detection of thrombin or cocaine. CNTs with pentacyaneferrate-modified chitosan nanocomposites on a glassy carbon electrode demonstrate bioelectrical catalytic oxidation, which can be monitored using cyclic voltammetry in a biosensor for glucose. A xanthine oxidase modified carbon film and multi-walled CNT/carbon film electrodes allowed the design of a new hypoxanthine biosensor [9]. Optical biosensors have also found various applications. In most cases, fluorescence is the measured signal; either the near-infrared fluorescence of semiconducting SWCNTs, for example, or their excellent quenching ability in comparison to conventional organic quenchers can be used. A promising application in the field of environmental analysis, especially for determining pesticides in water, is given in a review on the advances of pesticide biosensors. Additionally, the current status, challenges and future perspectives for CNTs are also demonstrated for both electrochemical and optical measurements [10]. Recently, biosensors in which the recognition elements are immobilised on a paper strip have gained increasing interest because of negligible costs for disposables. Known from dipsticks, such paper-based sensors have become the first choice for disposable sensors and integrated sensing platforms. Within a recent review article on sensing approaches on paper-based devices, the performance and application possibilities for modified paper material containing CNTs is discussed for electrochemical and optical read out [11]. CNTs have even found applications in the interesting use of molecular beacons [12].

Thus, a short survey of the literature demonstrates the increasing involvement of CNTs in a large number of applications to improve electrode qualities, for use as a recognition element, or even as part of the detection system. Such nanoparticles imply the development of further characterisation methods. Further research is essential to obtain more data and information on the properties of these CNTs, which are interesting for various applications, but are also toxic, either by themselves or through their modified cores. The journal Analytical and Bioanalytical Chemistry will continue to report on the analytical properties, characterisation techniques and necessary regulations to overcome disadvantages in the future, since, at present, these nanoparticles are used in cosmetics and nutrition. Only modern analytics can supply detailed knowledge of the properties of CNTs and safe applications in traditional, cosmetics and medical fields.

\section{References}

1. Valcárel M, Simonet BM, Cárdenas S, Suárz B (2005) Present and future applications of carbon nanotubes to analytical science. Anal Bioanal Chem 382:1783-1790

2. Polo-Luque ML, Simonet BM, Valcárcel M (2012) Coiled carbon nanotubes combined with ionic liquid: a new soft material for SPE. Anal Bioanal Chem 404:903-907 
3. Azzouz I, Vial J, Thiébaut D, Haudebourg R, Danaie K, Sassiat P, Breviere J (2014) Review of stationary phases for microelectromechanical systems in gas chromatography: feasibility and separations. Anal Bioanal Chem 406:981-994

4. Gonzáles-Curbelo MA, Asensio-Rames M, Herrera-Herrera AV, Hernández-Borges J (2012) Pesticide residue analysis in cereal-based baby foods using multi-walled carbon nanotubes dispersive solid-phase extraction. Anal Bioanal Chem 404: 183-196

5. Hussain CM, Mitra S (2011) Micropreconcentration units based on carbon nanotubes (CNT). Anal Bioanal Chem 399: 75-89

6. Saito N, Haniu H, Usui Y, Aoki K, Hara K, Takanashi S, Shimizu M, Narita N, Okamoto M, Kobayashi Shinsuke et al (2014) Safe clinical use of carbon nanotubes as innovative biomaterials. Chem Rev (Washington, DC, United States), Ahead of Print

7. Nelson DJ, Shagufta KR (2012) Characterization of a tamoxifen-tethered single-walled carbon nanotube conjugate by using NMR spectroscopy. Anal Bioanal Chem 404:771776

8. Cialla D, März A, Böhme R, Theil F, Weber K, Schmitt M, Popp J (2012) Surface-enhanced Raman spectroscopy (SERS): progress and trends. Anal Bioanal Chem 403:27-54

9. Torres AC, Ghica ME, Brett CMA (2013) Design of a new hypoxanthine biosensor: xanthine oxidase modified carbon film and multi-walled carbon nanotube/carbon film electrodes. Anal Bioanal Chem 405:3813-3822
10. Liu SQ, Zheng ZZ, Li XY (2013) Advances in pesticide biosensors: current status, challenges, and future perspectives. Anal Bioanal Chem 405:63-90

11. Nery EW, Kubota LT (2013) Sensing approaches on paper-based devices: a review. Anal Bioanal Chem 405:7573-7595

12. Huang K, Marti AA (2012) Recent trends in molecular beacon design and applications. Anal Bioanal Chem 402:3091-3102

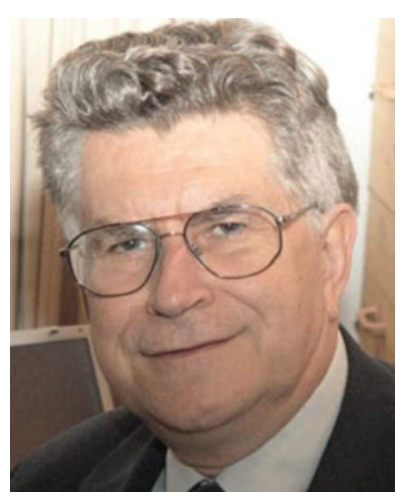

Günter Gauglitz is Senior Professor at the Eberhard-KarlsUniversity of Tübingen working in analytical and physical chemistry. He was chairman of the GDCh Division of Analytical Chemistry and chaired the Europt(r)ode VIII meeting. For more than 15 years his main scientific interests have centered on research and development in chemical and biochemical sensors with special focus on the characterization of interfaces of polymers and biomembrane surfaces, spectroscopic techniques, use of spectral interferometry to monitor changes in optical thickness of thin layers, and effects of Fresnel reflectivity at interfaces. He has been Editor of Analytical and Bioanalytical Chemistry (ABC) since 2002. 